\title{
Existence of Positive Solutions for a Fourth-Order Three-Point BVP with Sign-Changing Green's Function
}

\author{
Alhussein Mohamed \\ College of Mathematics and Statistics, Northwest Normal University, Lanzhou, China \\ Email: tethussain60@gmail.com
}

How to cite this paper: Mohamed, A. (2021) Existence of Positive Solutions for a Fourth-Order Three-Point BVP with Sign-Changing Green's Function. Applied Mathematics, 12, 311-321. https://doi.org/10.4236/am.2021.124022

Received: March 16, 2021

Accepted: April 19, 2021

Published: April 22, 2021

Copyright $\odot 2021$ by author(s) and Scientific Research Publishing Inc. This work is licensed under the Creative Commons Attribution International License (CC BY 4.0).

http://creativecommons.org/licenses/by/4.0/

\section{(c) (i) Open Access}

\section{Abstract}

In this article, by using a fixed point theorem, we study following fourth-order three-point BVP: $\left\{\begin{array}{l}u^{(4)}(t)=\lambda f(t, u(t)), \quad t \in[0,1], \\ u^{\prime}(0)=u^{\prime \prime}(0)=u(1)=0, \\ \alpha u(0)+u^{\prime \prime \prime}(\eta)=0\end{array}\right.$ where $f \in C([0,1] \times[0,+\infty),[0,+\infty)) \quad \alpha \in[0,6)$ and $\eta \in\left[\frac{1}{2}, 1\right)$. The main point to emphasize is that although the corresponding Green's function is changing signs, by applying the fixed point theorem, we can still obtain at least two positive solutions and degreased solutions under certain suitable conditions.

\section{Keywords}

Fourth-Order Fourth-Point Boundary Value Problem, Function, Positive Solution, Existence, Fixed Point Greens Function

\section{Introduction}

The boundary value problem of fourth-order ordinary differential equations (BVP for short) has attracted much attention due to its amazing application in engineering, physics, material mechanics, fluid mechanics and so on. Many authors use Banach contraction to study the existence of single or multiple positive solutions for certain third-order BVP-Guo (Orem), Guo-Krasnoselsky (Krasnoselsky) Fixed point theorem, Leray-Schauder nonlinear substitution, fixed point index theory of viewing cone, monotonic iterative technique, upper and lower solution method, degree theory, the Critical point theorem in a conical shell, etc. see [1]-[6]. 
However, it is necessary to point out that, in most of the existing literature, the Greens functions involved are nonnegative, which is an important condition in the study on BVP Positive Solution.

Recently, when the corresponding Green's function is changing signs, some work has been done on the positive solution of the second or third order BVP. For example, Zhong and An [7] studied the existence of at least one positive solution of the following second-order periodic BVP with positive and negative transformation Green's function

$$
\left\{\begin{array}{l}
u^{(3)}(t)+\rho^{(2)} u=\lambda f(u), \quad 0<t<T, \\
u(0)=u(T), \\
u^{\prime}(0)=u^{\prime}(T) .
\end{array}\right.
$$

where $0<\rho \leq \frac{3 \Pi}{2 T}$. The main tool used is the fixed point index theory of cone mapping 2008, for a singular third-order three-point BVP of Green's function with infinite signature

$$
\left\{\begin{array}{l}
u^{(3)}(t)=a(t) f(t, u(t)), \quad 0<t<1 \\
u(0)=u(1)=u^{\prime \prime}(\eta)=0
\end{array}\right.
$$

where $\eta \in\left(\frac{17}{24}, 1\right)$. Palamide and Smirlis [8] discussed the existence of at least one positive solution. Their technique is a combination of Guo-Krasnosel'sski fixed point theory and the corresponding vector field characteristics. In 2012, Sun and Zhao [9] [10] obtained single or multiple positive solutions with three-point positive and negative BVP by applying the fixed point theory of Guo-Krasnosel'skii and Leggett-Williams.

$$
\left\{\begin{array}{l}
u^{(3)}(t)=f(t, u(t)), \quad 0<t<1 \\
u(0)=u(1)=u^{\prime \prime}(\eta)=0
\end{array}\right.
$$

where $\eta \in\left(\frac{1}{2}, 1\right)$. For relevant results, one can refer to [11]-[18]. It is worth mentioning that there are other types of achievements on either sign-changing or vanishing Green's functions which prove the existence of sign-changing solutions, positive in some cases, see [11] [19] [20] [21] [22].

Inspired and inspired by the above works, this article focuses on the following fourth-order three-point BVP with the iconic Green's function.

$$
\left\{\begin{array}{l}
u^{(4)}(t)=\lambda f(t, u(t)), \quad t \in[0,1], \\
u^{\prime}(0)=u^{\prime \prime}=u(1)=0, \\
\alpha u(0)+u^{\prime \prime \prime}(\eta)=0
\end{array}\right.
$$

Throughout this paper, we always assume that $\alpha \in[0,6)$ and $\eta \in\left[\frac{1}{2}, 1\right)$. Obviously, the BVP (2.1) is a special case of the BVP (2.2). However, it is neces- 
sary to point out that this paper is not a simple extension of [23], which is different from the restriction in [23]. On the other hand, compared with [23], we can only prove that the obtained solution is concave on $[0, \eta]$.

Our main tool is the following well-known Guo-Krasnoselskii fixed point theorem [24] [25]:

Let $K$ be a cone in a real Banach space $E$.

Definition 1.1. A functional $\rho: K \rightarrow R$ is said to be increasing on $K$ provided $\rho(x) \leq \rho(y)$ for all $x, y \in K$ with $x \leq y$, where $x \leq y$ if and only if $y-x \in K$.

Definition 1.2. Let $\phi: K \rightarrow[0,+\infty)$ be continuous. For each $d>0$, one defines the set

$$
K(\phi, d)=\{u \in K: \phi(u)<d\}
$$

Theorem 1.1. Let $\rho$ and $\phi$ be increasing, nonnegative, and continuous functionals on $K$, and let $\varphi$ be a nonnegative continuous functional on $K$ with $\varphi(0)=0$ such that, for some $c>0$ and $M>0$,

$$
\phi(u) \leq \varphi(u) \leq \rho(u),\|u\| \leq M \phi(u)
$$

for all $u \in K(\phi, c)$. Suppose there exist a completely continuous operator $T: K(\phi, c) \rightarrow K$ and $0<a<b<c$ such that

$$
\varphi(\xi u) \leq \xi \varphi(u) \text { for } 0 \leq \xi \leq 1, u \in \partial K(\varphi, b),
$$

and

(H1) $\phi(T u)>c$ for all $u \in \partial K(\phi, c)$;

(H1) $\varphi(T u)<b c$ for all $u \in \partial K(\varphi, b)$;

(H3) $K(\rho, a) \neq 0$ and $\rho(T u)>a$ for all $u \in \partial K(\rho, a)$.

Then $T$ has at least two fixed points $u^{*}$ and $u^{* * *}$ in $K(\phi, c)$ such that

$$
\begin{aligned}
& a<\rho\left(u^{*}\right) \text { with } \varphi\left(u^{*}\right)<b, \\
& b<\varphi\left(u^{* *}\right) \text { with } \phi\left(u^{* *}\right)<c .
\end{aligned}
$$

\section{Preliminaries}

The remainder of this paper, we assume that Banach space $C[0,1]$ is equipped with the norm $\|u\|=\max _{t \in[0,1]}|u(t)|$.

For the following BVP:

$$
\left\{\begin{array}{l}
u^{(4)}(t)=\lambda f(t, u(t)), \quad t \in[0,1], \\
u^{\prime}(0)=u^{\prime \prime}(0)=u(1)=0, \\
\alpha u(0)+u^{\prime \prime \prime}(\eta)=0
\end{array}\right.
$$

then we have the following lemma.

Lemma 2.1. The BVP (2.1) has only trivial solution.

Proof. Easy to check.

Now, for any $y \in C[0,1]$, we consider the boundary value problems 


$$
\left\{\begin{array}{l}
u^{(4)}(t)=\lambda f(t, u(t)), \quad t \in[0,1] \\
u^{\prime}(0)=u^{\prime \prime}(0)=u(1)=0, \\
\alpha u(0)+u^{\prime \prime \prime}(\eta)=0
\end{array}\right.
$$

After a direct computation, one may obtain the expression of Green's function $G(t, s)$ of the BVP (2) as following:

For $s \geq \eta$

$$
G(t, s)=\left\{\begin{array}{l}
\frac{-\left(1-\alpha t^{3}\right)(1-s)^{3}}{6(6-\alpha)} \quad 0 \leq t \leq s, \\
\frac{(t-s)^{3}}{6}-\frac{\left(1-\alpha t^{3}\right)(1-s)^{3}}{6(6-\alpha)} \quad s \leq t \leq 1
\end{array}\right.
$$

and $s<\eta$

$$
G(t, s)=\left\{\begin{array}{l}
\frac{6\left(1-t^{3}\right)-\left(1-\alpha t^{3}\right)(1-s)^{3}}{6(6-\alpha)} \quad 0 \leq t \leq s, \\
\frac{(t-s)^{3}}{6}+\frac{6\left(1-t^{3}\right)-\left(1-\alpha t^{3}\right)(1-s)^{3}}{6(6-\alpha)} \quad s \leq t \leq 1
\end{array}\right.
$$

Lemma 2.2. It is not difficult to verify that $G(t, s)$ has the following characteristics:

1) If $s \in[1, \eta]$, then $G(t, s)$ is nonincreasing with respect to $t \in[0,1]$.

2) $G(t, s)$ changes its sign on $[0,1] \times[0,1]$. In details, if $(t, s) \in[0,1] \times[0, \eta]$, then $G(t, s) \geq 0$. If $(t, s) \in[0,1] \times[1, \eta]$, then $G(t, s) \leq 0$.

3) If $s \geq \eta$, then $\max _{t \in[0,1]} G(t, s)=G(1, s)=0$ such that

$$
G(t, s) \geq 0 \text { for } 0 \leq s \leq \eta \text { and } G(t, s) \leq 0 \text { for } \eta \leq s \leq 1 .
$$

Moreover, if $s \geq \eta$, then

$$
\begin{gathered}
\max G(t, s): t \in[0,1]=G(1, s)=0, \\
\min G(t, s): t \in[0,1]=G(0, s)=\frac{-(1-s)^{3}}{6(6-\alpha)}
\end{gathered}
$$

if $s<\eta$, then

$$
\begin{gathered}
\max G(t, s): t \in[0,1]=G(0, s)=\frac{6-(1-s)^{3}}{(6-\alpha)}, \\
\min G(t, s): t \in[0,1]=G(1, s)=0
\end{gathered}
$$

Now, let $K_{0}=\{y \in C[0,1]: y(t)\}$ is nonnegative and decreasing on $[0,1]$.

Then $K_{0}$ is a cone in $\mathrm{C}[0,1]$.

Lemma 2.3. Let $y \in K_{0}$ and $u(t)=\int_{0}^{1} G(t, s) y(s) \mathrm{d} s, t \in[0,1]$. Then $u$ is the unique solution of the BVP (1.2) and $u \in K_{0}$. Moreover, $u(t)$ is concave on $[0, \eta]$.

Proof. For $0 \leq t \leq \eta$, we have 


$$
\begin{aligned}
u(t) & =\int_{0}^{t}\left[\frac{(t-s)^{3}}{6}+\frac{6\left(1-t^{3}\right)-\left(1-\alpha t^{3}\right)(1-s)^{3}}{6(6-\alpha)}\right] y(s) \mathrm{d} s \\
& +\int_{t}^{\eta}\left[\frac{6\left(1-t^{3}\right)-\left(1-\alpha t^{3}\right)(1-s)^{3}}{6(6-\alpha)}\right] y(s) \mathrm{d} s \\
& +\int_{\eta}^{1} \frac{-\left(1-\alpha t^{3}\right)(1-s)^{3}}{6(6-\alpha)} y(s) \mathrm{d} s
\end{aligned}
$$

since $\eta \geq \frac{1}{2}$ we get

$$
\begin{aligned}
u^{\prime}(t)= & \frac{\alpha t^{2}}{2(6-\alpha)} \int_{0}^{\eta}\left[(1-s)^{3}-1\right] y(s) \mathrm{d} s+\frac{1}{2} \int_{0}^{t}\left(s^{2}-2 t s\right) y(s) \mathrm{d} s \\
& -\frac{t^{2}}{2} \int_{t}^{\eta} y(s) \mathrm{d} s+\int_{\eta}^{1} \frac{\alpha t^{2}(1-s)^{3}}{2(6-\alpha)} y(s) \mathrm{d} s \\
\leq & y(\eta)\left[\frac{\alpha t^{2}}{2(6-\alpha)} \int_{0}^{\eta}\left[(1-s)^{3}-1\right] \mathrm{d} s+\frac{1}{2} \int_{0}^{t}\left(s^{2}-2 t s\right) \mathrm{d} s\right. \\
& \left.-\frac{t^{2}}{2} \int_{t}^{\eta} \mathrm{d} s+\int_{\eta}^{1} \frac{\alpha t^{2}(1-s)^{3}}{2(6-\alpha)} \mathrm{d} s\right] \\
& \leq t^{2} y(\eta)\left[\frac{\alpha(1-4 \eta)}{8(6-\alpha)}-\frac{\eta}{2}+\frac{t}{6}\right] \leq t^{2} y(\eta)\left[\frac{\alpha(1-4 \eta)}{8(6-\alpha)}+\frac{\eta}{3}\right] \leq 0
\end{aligned}
$$

At the same time, $\eta>\frac{1}{2}$ shows that

$$
\begin{aligned}
u^{\prime \prime}(t) & =\frac{\alpha t}{(6-\alpha)} \int_{0}^{\eta}\left[(1-s)^{3}-1\right] y(s) \mathrm{d} s-\int_{0}^{t} s y(s) \mathrm{d} s \\
& -t \int_{t}^{\eta} y(s) \mathrm{d} s+\int_{\eta}^{1} \frac{\alpha t(1-s)^{3}}{(6-\alpha)} y(s) \mathrm{d} s \\
\leq & y(\eta)\left[\frac{\alpha t}{(6-\alpha)} \int_{0}^{\eta}\left[(1-s)^{3}-1\right] \mathrm{d} s-\int_{0}^{t} s \mathrm{~d} s-t \int_{t}^{\eta} \mathrm{d} s+\int_{\eta}^{1} \frac{\alpha t(1-s)^{3}}{(6-\alpha)} \mathrm{d} s\right] \\
& \leq t y(\eta)\left[\frac{\alpha(1-4 \eta)}{4(6-\alpha)}-\eta+\frac{t}{2}\right] \leq t y(\eta)\left[\frac{\alpha(1-4 \eta)}{4(6-\alpha)}-\frac{\eta}{2}\right] \leq 0 \quad t \in(0, \eta)
\end{aligned}
$$

For $t \in(\eta, 1)$, we have

$$
\begin{aligned}
u(t)= & \int_{0}^{\eta}\left[\frac{(t-s)^{3}}{6}+\frac{6\left(1-t^{3}\right)-\left(1-\alpha t^{3}\right)(1-s)^{3}}{6(6-\alpha)}\right] y(s) \mathrm{d} s \\
& +\int_{\eta}^{t}\left[\frac{(t-s)^{3}}{6}-\frac{\left(1-\alpha t^{3}\right)(1-s)^{3}}{6(6-\alpha)}\right] y(s) \mathrm{d} s \\
& +\int_{t}^{1}\left[\frac{-\left(1-\alpha t^{3}\right)(1-s)^{3}}{6(6-\alpha)}\right] y(s) \mathrm{d} s
\end{aligned}
$$

In view of $y \in K_{0}$ and $\eta>\frac{1}{2}$, we get 


$$
\begin{aligned}
u^{\prime}(t)= & \frac{\alpha t^{2}}{2(6-\alpha)} \int_{0}^{\eta}\left[(1-s)^{3}-1\right] y(s) \mathrm{d} s+\frac{1}{2} \int_{0}^{\eta}\left(s^{2}-2 t s\right) y(s) \mathrm{d} s \\
& +\int_{\eta}^{t} \frac{(t-s)^{2}}{2}+\int_{\eta}^{1} \frac{\alpha t^{2}(1-s)^{3}}{2(6-\alpha)} y(s) \mathrm{d} s \\
\leq & y(\eta)\left[\frac{\alpha t^{2}}{2(6-\alpha)} \int_{0}^{\eta}\left[(1-s)^{3}-1\right] \mathrm{d} s+\frac{1}{2} \int_{0}^{\eta}\left(s^{2}-2 t s\right) \mathrm{d} s\right. \\
& \left.+\int_{\eta}^{t} \frac{(t-s)^{2}}{2}+\int_{\eta}^{1} \frac{\alpha t^{2}(1-s)^{3}}{2(6-\alpha)} \mathrm{d} s\right] \\
= & t^{2} y(\eta)\left[\frac{-\alpha \eta}{2(6-\alpha)}+\frac{t}{6}-\frac{\eta}{2}\right] \leq t^{2} y(\eta)\left[\frac{-\alpha \eta}{2(6-\alpha)}-\frac{\eta}{3}\right] \leq 0 \quad t \in(\eta, 1)
\end{aligned}
$$

Obviously, $u^{\prime \prime \prime}(t)=y(t)$ for $t \in[0,1], u^{\prime}(0)=u^{\prime \prime \prime}(0)=u(1)=0$,

$\alpha u(0)+u^{\prime \prime}(\eta)=0$. This shows that $u$ is a solution of the BVP (2.2). The uniqueness follows immediately from Lemma 2.1. Since $u^{\prime}(t) \leq 0$ for $t \in[0,1]$ and $u(1)=0$, we have $u(t) \geq 0$ for $t \in[0,1]$. So, $u \in K_{0}$. In view of $u^{\prime \prime}(t) \leq 0$ for $t \in[0, \eta]$, we know that $u(t)$ is concave on $[0, \eta]$.

Lemma 2.4. Assume $y \in K_{0}$ then the unique solution $u(t)$ of the BVP (2.2) satisfies

$$
\min _{t \in[0, \mu]} u(t) \geq \mu^{*}\|u\|
$$

where $\mu \in\left(0, \frac{1}{2}\right]$ and $\mu^{*}=\frac{\eta-t}{\eta}$.

Proof. From Lemma 2.2, we know that $u(t)$ is concave on $[0, \eta]$, thus for $t \in[0, \eta]$,

$$
u(t) \geq \frac{\eta-t}{\eta} u(0)+\frac{t}{\eta} u(\eta)
$$

In view of $u \in K_{0}$, we know that $\|u\|=u(0)$, which together with (2.3) implies that

$$
u(t) \geq \frac{\eta-t}{\eta}\|u\|, \quad 0 \leq t \leq \eta
$$

according to that

$$
\min _{t \in[0, \mu]} u(t)=u(\mu) \geq \frac{\eta-\mu}{\eta}\|u\|=\mu^{*}\|u\|
$$

\section{Main Results}

In this section, we are concerned with the existence of at least one positive solution of the problem (2.1). Assume that

(C1) For each $u \in[0,+\infty)$, the mapping $t \mapsto f(t, u)$ is decreasing;

(C2) For each $t \in[0,1]$, the mapping $u \mapsto f(t, u)$ is increasing.

Let

$$
K=\left\{u \in K_{0}: \min _{t \in[0, \mu]} u(t) \geq \mu^{*}\|u\|\right\}
$$


Then it is easy to see that $K$ is a cone in $C[0,1]$.

Now, we define an operator $A: K \rightarrow K$ by

$$
(A u)(t)=\int_{0}^{1} G(t, s) f(s, u(s)) \mathrm{d} s, t \in[0,1]
$$

distinctly, if $u$ is a fixed point of $A$ in $K$, then $u$ is a positive and nondecreasing solution of the BVP (2.2), by lemma 2.3 and lemma 2.4 we know, $A: K \rightarrow K$ although $G(t, s)$ is not continuous, it follows from known textbook results, for example, see [26], that $A: K \rightarrow K$, is completely continuous. Set

$$
\mu^{*}=\frac{\eta-\mu}{\eta}, \quad P=\int_{0}^{\eta} \frac{1-(1-s)^{3}}{(6-\alpha)} \mathrm{d} s \quad \text { and } \quad Q=\int_{0}^{\mu} G(\eta, s) \mathrm{d} s
$$

Lemma 3.1. Suppose that (C1) and (C2) hold. Moreover, If there exist three constants $a, b$ and $c$ with $0<a<b<\mu^{*} c$ such that

(F1) $f(\mu, c)>\frac{c}{P}$,

(F2) $f\left(0, \frac{b}{\mu^{*}}\right)<\frac{b}{Q}$,

(F3) $f\left(\mu, \mu^{*} a\right)>\frac{a}{P}$

then boundary value problem (1.1) has at least two positive solutions $u \in K$.

Proof. First, we define the increasing, nonnegative, and continuous functionals $\phi, \varphi$ and $\rho$ on $K$ as follows:

$$
\begin{gathered}
\phi(u)=\min _{t \in[0, \mu]} u(t)=u(\mu), \\
\varphi(u)=\max _{t \in[\mu, 1]} u(t)=u(\mu), \\
\rho(u)=\max _{t \in[0,1]} u(t)=u(0) .
\end{gathered}
$$

Obviously, for any $u \in K, \phi(u)=\varphi(u) \leq \rho(u)$. At the same time, for each $u \in K$, in view of $\phi(u)=\min _{t \in[0, \mu]} u(t) \geq \mu \in\|u\|$, we have

$$
\|u\| \leq \frac{1}{\mu^{*}} \phi(u) \quad \text { for } u \in K .
$$

Furthermore, we also note that $\varphi(\xi u)=\xi \varphi(u)$ for $0 \leq \xi \leq 1, u \in K$.

Next, for any $u \in K$, we claim that

$$
\int_{\mu}^{1} G(\eta, s) f(s, u(s)) \mathrm{d} s \geq 0
$$

In fact, it follows from (C1), (C2), and $\eta \geq \frac{\alpha+4}{16-\alpha}$

$$
\begin{aligned}
& \int_{\mu}^{1} G(\eta, s) f(s, u(s)) \mathrm{d} s \\
& =\int_{\mu}^{\eta} G(\eta, s) f(s, u(s)) \mathrm{d} s+\int_{\eta}^{1} G(\eta, s) f(s, u(s)) \mathrm{d} s \\
& \leq \int_{\mu}^{\eta}\left[\frac{(\eta-s)^{3}}{6}+\frac{6\left(1-\eta^{3}\right)-\left(1-\alpha \eta^{3}\right)(1-s)^{3}}{6(6-\alpha)}\right] y(s) \mathrm{d} s \\
& +\int_{\eta}^{1} \frac{-\left(1-\alpha t^{3}\right)(1-s)^{3}}{6(6-\alpha)} f(s, u(s)) \mathrm{d} s
\end{aligned}
$$




$$
\begin{aligned}
= & f(\eta, u(\eta))\left[\int_{\mu}^{\eta} \frac{(\eta-s)^{3}}{6}+\frac{6\left(1-\eta^{3}\right)-\left(1-\alpha \eta^{3}\right)(1-s)^{3}}{6(6-\alpha)} \mathrm{d} s\right. \\
& \left.+\int_{\eta}^{1} \frac{-\left(1-\alpha \eta^{3}\right)(1-s)^{3}}{6(6-\alpha)} \mathrm{d} s\right] \\
\geq & \frac{f(\eta, u(\eta))}{24(6-\alpha)}\left[\alpha \eta^{3}(1-\eta)+24 \eta-18 \eta^{4}-1+\mu\left(36 \eta^{2}+3 \alpha \eta^{3}-6 \alpha \eta^{2}-23\right)\right. \\
& \left.+\mu^{3} \eta(4 \alpha-24)+\mu^{4}(6-\alpha)\right] \\
= & \frac{f(\eta, u(\eta))}{24(6-\alpha)}\left[\alpha \eta^{3}(1-\eta)+24 \eta-18 \eta^{4}+10 \eta^{2}+\eta^{3}-\frac{20}{27}-\frac{697}{81}\right] \geq 0,
\end{aligned}
$$

Now, we assert that $\phi(A u)>c$ for all $u \in \partial K(\phi, c)$. To prove this, let $u \in \partial K(\phi, c)$; that is, $u \in K$ and $\phi(u)=u(\mu)=c$. Then

$$
u(t) \geq u(\mu)=c, t \in[0, \mu] .
$$

Since $(A u)(t)$ is decreasing on $[0,1]$, it follows from (3.1), (3.2), (C2), (C1) and (F1) that

$$
\begin{aligned}
\phi(A u) & =(A u)(\mu) \geq(A u)(\eta)=\int_{0}^{1} G(\eta, s) f(s, u(s)) \mathrm{d} s \\
& \geq \int_{0}^{\mu} G(\eta, s) f(s, u(s)) \mathrm{d} s \geq \int_{0}^{\mu} G(\eta, s) f(\mu, c) \mathrm{d} s \\
& >\frac{c}{P} \int_{0}^{\mu} G(\eta, s) \mathrm{d} s=c .
\end{aligned}
$$

Then, we assert that $\varphi(A u)<b$ for all $u \in \partial K(\varphi, b)$. To see this, suppose that $u \in \partial K(\varphi, b)$; that is, $u \in K$ and $\varphi(u)=b$. Since $\|u\| \leq \frac{1}{\mu^{*}} \phi(u)=\frac{1}{\mu^{*}} \varphi(u)$, we have

$$
0 \leq u(t) \leq\|u\| \leq \frac{b}{\mu^{*}}, t \in[0, \eta]
$$

In view of the properties of $G(t, s),(\mathrm{F} 2),(3.3),(\mathrm{C} 1)$ and (C2), we get

$$
\begin{aligned}
\varphi(A u) & =(A u)=\int_{0}^{1} G(\eta, s) f(s, u(s)) \mathrm{d} s \leq \int_{0}^{\eta} G(\mu, s) f(s, u(s)) \mathrm{d} s \\
& \leq \int_{0}^{\eta} G(\mu, s) f\left(0, \frac{b}{\mu^{*}}\right) \mathrm{d} s<\frac{b}{Q} \int_{0}^{\eta} G(\mu, s) \mathrm{d} s=b .
\end{aligned}
$$

Finally, we assert that $K(\rho, a) \neq 0$ and $\rho(A u)>a$ for all $u \in \partial K(\rho, a)$. In fact, the constant function $\frac{a}{2} \in K(\rho, a)$. Moreover, for $u \in \partial K(\rho, a)$, that is $u \in K$ and $\rho(u)=u(0)=a$. Then

$$
u(t) \geq \mu^{*}\|u\|=\mu^{*} u(0)=\mu^{*} a, t \in[0, \mu] .
$$

Since $(A u)(t)$ is decreasing on $[0,1]$, it follows from (F3), (3.1), (3.4), (C1) and $(\mathrm{C} 2)$ that

$$
\rho(A u)=(A u)(0) \geq(A u)(\eta)=\int_{0}^{1} G(\eta, s) f(s, u(s)) \mathrm{d} s
$$




$$
\begin{aligned}
& \geq \int_{0}^{\mu} G(\eta, s) f(s, u(s)) \mathrm{d} s \geq \int_{0}^{\mu} G(\eta, s) f\left(\mu, \mu^{*} a\right) \mathrm{d} s \\
& >\frac{a}{P} \int_{0}^{\mu} G(\eta, s) \mathrm{d} s=a .
\end{aligned}
$$

To sum up, all the hypotheses of Theorem 1.1 are satisfied. Consequently $A$ has at least two fixed points; that is, the BVP (1.1) has at least two positive solutions $u^{*}$ and $u^{* *}$ such that

$$
\begin{aligned}
& a<\max _{t \in[0,1]} u^{*}(t) \text { with } \max _{t \in[\mu, 1]} u^{*}(t)<b \\
& b<\max _{t \in[\mu, 1]} u^{* *}(t) \text { with } \min _{t \in[0, \mu]} u^{* *}(t)<c .
\end{aligned}
$$

\section{Acknowledgements}

The author expresses gratitude to the referees for their valuable comments and suggestions.

\section{The Term of the Foundation}

This work is partly supported by the National Natural Science Foundation of China (11561064) and partly supported by NWNU-LKQN-14-6.

\section{Conflicts of Interest}

The author declares that there is no conflict of interest with the publication of this article.

\section{References}

[1] Yao, Q.L. (2003) The Existence and Multiplicity of Positive Solutions for a Third-Order Three-Point Boundary Value Problem. Acta Mathematicae Applicatae Sinica, 19, 117-122. https://doi.org/10.1007/s10255-003-0087-1

[2] Sun, Y.P. (2005) Positive Solutions of Singular Third-Order Three-Point Boundary Value Problem. Journal of Mathematical Analysis and Applications, 306, 589-603. https://doi.org/10.1016/j.jmaa.2004.10.029

[3] Palamides, A.P. and Stavrakakis, N.M. (2010) Existence and Uniqueness of a Positive Solution for a Third-Order Three-Point Boundary-Value Problem. Electronic Journal of Differential Equations, 2010, Paper No. 155.

[4] Guo, L.-J., Sun, J.-P. and Zhao, Y.-H. (2008) Existence of Positive Solutions for Nonlinear Third-Order Three-Point Boundary Value Problems. Nonlinear Analysis: Theory, Methods Applications, 68, 3151-3158. https://doi.org/10.1016/j.na.2007.03.008

[5] Bai, Z.B. and Fei, X.L. (2006) Existence of Triple Positive Solutions for a Third Order Generalized Right Focal Problem. Mathematical Inequalities \& Applications, 9 , 437-444. https://doi.org/10.7153/mia-09-42

[6] Anderson, D.R. (2003) Green's Function for a Third-Order Generalized Right Focal Problem. Journal of Mathematical Analysis and Applications, 288, 1-14. https://doi.org/10.1016/S0022-247X(03)00132-X

[7] Zhong, S. and An, Y. (2011) Existence of Positive Solutions to Periodic Boundary Value Problems with Sign-Changing Green's Function. Boundary Value Problems, 
2011, Article No. 8. https://doi.org/10.1186/1687-2770-2011-8

[8] Palamides, A.P. and Smyrlis, G. (2008) Positive Solutions to a Singular Third-Order Three-Point Boundary Value Problem with an Indefinitely Signed Green's Function. Nonlinear Analysis. Theory, Methods \& Applications, 68, 2104-2118. https://doi.org/10.1016/j.na.2007.01.045

[9] Sun, J.-P. and Zhao, J. (2012) Positive Solution for a Third-Order Three-Point Boundary Value Problem with Sign-Changing Green's Function. Communications in Applied Analysis, 16, 219-228. https://doi.org/10.1186/1687-2770-2012-12

[10] Sun, J.-P. and Zhao, J. (2012) Multiple Positive Solutions for a Third-Order Three-Point BVP with Sign-Changing Green's Function. Electronic Journal of Differential Equations, 2012, No. 118.

[11] Zhao, Y.-H. and Li, X.-L. (2014) Iteration for a Third-Order Three-Point BVP with Sign-Changing Green's Function. Journal of Applied Mathematics, 2014, Article ID: 541234. https://doi.org/10.1155/2014/541234

[12] Palamides, A.P., Veloni, A. and Alatsathianos, S. (2013) Positive Solutions of a Third-Order BVP Independent of the Sign of the Green's Function. Differential Equations and Dynamical Systems, 21, 261-279. https://doi.org/10.1007/s12591-012-0151-5

[13] Gao, C., Zhang, F. and Ma, R. (2017) Existence of Positive Solutions of Second-Order Periodic Boundary Value Problems with Sign-Changing Green's Function. Acta Mathematicae Applicatae Sinica, English Series, 33, 263-268.

https://doi.org/10.1007/s10255-017-0657-2

[14] Gao, L.-J. and Sun, J.-P. (2014) Positive Solutions of a Third-Order Three-Point BVP with Sign-Changing Green's Function. Mathematical Problems in Engineering, 2014, Article ID: 406815. https://doi.org/10.1155/2014/406815

[15] Li, X.-L., Sun, J.-P. and Kong, F.-D. (2013) Existence of Positive Solution for a Third-Order Three-Point BVP with Sign-Changing Green's Function. Electronic Journal of Qualitative Theory of Differential Equations, 2013, No. 30.

[16] Ma, R. (2011) Nonlinear Periodic Boundary Value Problems with Sign-Changing Green's Function. Nonlinear Analysis. Theory, Methods \& Applications, 74, 1714-1720. https://doi.org/10.1016/j.na.2010.10.043

[17] Niu, B.-W., Sun, J.-P. and Ren, Q.-Y. (2015) Two Positive Solutions of Third-Order BVP with Integral Boundary Condition and Sign-Changing Green's Function. Journal of Function Spaces, 2015, Article ID: 491423. https://doi.org/10.1155/2015/491423

[18] Sun, J.-P. and Zhao, J. (2013) Iterative Technique for a Third-Order Three-Point BVP with Sign-Changing Green's Function. Electronic Journal of Differential Equations, 2013, No. 215.

[19] Cabada, A., Enguica, R. and Lopez-Somoza, L. (2017) Positive Solutions for Second-Order Boundary-Value Problems with Sign Changing Green's Functions. Electronic Journal of Differential Equations, 2017, No. 245.

[20] Infante, G. and Webb, J.R.L. (2006) Loss of Positivity in a Nonlinear Scalar Heat Equation. Nonlinear Differential Equations and Applications, 13, 249-261. https://doi.org/10.1007/s00030-005-0039-y

[21] Infante, G. and Webb, J.R.L. (2003) Three-Point Boundary Value Problems with Solutions That Change Sign. Journal of Integral Equations and Applications, 15, 37-57. https://doi.org/10.1216/jiea/1181074944

[22] Minhós, F. (2017) Solvability of Generalized Hammerstein Integral Equations on 
Unbounded Domains, with Sign-Changing Kernels. Applied Mathematics Letters, 65, 113-117. https://doi.org/10.1016/j.aml.2016.10.012

[23] Li, X.L., Sun, J.P. and Kong, F.D. (2013) Existence of Positive Solution for a Third-Order Three-Point BVP with Sign-Changing Green's Function. Electronic Journal of Qualitative Theory of Differential Equations, No. 30, 1-11.

[24] Guo, D.J. and Lakshmikantham, V. (1988) Nonlinear Problems in Abstract Cones. Academic Press, New York.

[25] Krasnoselskij, M.A. (1964) Positive Solutions of Operator Equations. Noordhoff, Groningen.

[26] Martin, R.H. (1976) Nonlinear Operators and Differential Equations in Banach Spaces. John Wiley and Sons, New York. 